\title{
PERTENECER A ALGO MAYOR: EXPERIENCIAS DE PACIENTES Y CUIDADORES DURANTE EL CUIDADO PALIATIVO EN CHILE
}

\author{
BELONGING TO SOMETHING GREATER: EXPERIENCES \\ OF PATIENTS AND CAREGIVERS ENROLLED IN PALLIATIVE CARE \\ SERVICES IN CHILE
}

\author{
Pablo Arriaza* \\ Guillermina CANCINO** \\ Olivia SanhueZa ${ }^{* * *}$
}

\begin{abstract}
RESUMEN
Estudio cualitativo realizado a fin de comprender el significado de las experiencias de los pacientes y los que les brindan cuidados durante el período que permanecen en el Programa de Cuidados Paliativos, así como las diferentes maneras de enfrentar su enfermedad terminal en Chile. Los resultados de este estudio señalan la importancia de encontrar un refugio, de lo contrario es o será difícil enfrentar las cosas; tal situación fue denominada "pertenecer a algo mayor". Estos resultados tienen implicancias hacia las áreas de investigación, práctica y para la elaboración de políticas públicas. Este estudio puede servir como motivación para futuras investigaciones que puedan enfocarse en áreas relacionadas con el tratamiento paliativo y el aspecto psicosocial del paciente $y / o$ familia.
\end{abstract}

Palabras clave: Significado de la experiencia paliativa, cáncer avanzado, cuidadores, programa cuidados paliativos, estudio cualitativo.

\begin{abstract}
The goal of this qualitative study was to understand the meaning of the experiences of patients and their caregivers enrolled in palliative care and facing a terminal illness in Chile. Two focus groups were conducted to understand the patients' experiences and the caregivers' experiences. Results point to the importance of finding a sense of refuge in order to cope with a terminal illness. The overarching theme of this study "belonging to something greater" emerged from the participants' experiences. The findings provide guidance to focus on further research, practice, and policy efforts in the area of palliative care in Chile. This study may serve as motivation to enhance research studies focusing on different bio-psychosocial aspects of palliative care and how these factors influence patients and/or families.
\end{abstract}

Key words: Meaning of palliative care experiences, advanced cancer, caregivers, palliative care program, qualitative study.

\footnotetext{
* Dr. en Trabajo Social, Prof. titular de Trabajo Social, Universidad de New Hampshire, EEUU. Trabajador Social Clínico, Licenciado. Email: pabloarriaza@gmail.com

** Trabajadora Social, Magíster en Trabajo Social, mención en Intervención Familiar, Instituto Nacional del Cáncer, Santiago, Chile. Email: naranjita1957@yahoo.com

${ }_{\star * \star}$ Dra. en Enfermería, Prof. titular Dpto. de Enfermería, Universidad de Concepción, Chile. Email: osanhue@udec.cl
} 


\section{INTRODUCCIÓN}

Los esfuerzos gubernamentales en Chile respecto de las personas con confirmación diagnóstica de cáncer terminal, han enfocado su atención en seguir las reglas de cuidados paliativos dictadas por la Organización Mundial de la Salud (1). En efecto, el año 1994 nace el Programa Nacional de Alivio del Dolor por Cáncer y Cuidados Paliativos (2), derivado del Programa Nacional de Cáncer del Ministerio de Salud, haciendo suyo el Modelo de Continuidad de los cuidados, propuesto por el programa de lucha contra el cáncer de la Organización Mundial de la Salud.

El cáncer terminal se entiende como una enfermedad oncológica avanzada y en progresión, sin evidencia clínica de respuesta hacía la remisión completa -curación o mejoría-, asociada a numerosos síntomas. Se consideran los cuidados paliativos como la asistencia al paciente y a su entorno por un equipo multiprofesional, con el principal objetivo de controlar los síntomas, entre ellos el dolor, para mejorar la calidad de vida del paciente y su familia (2).

En abril del año 2003, el Programa de Alivio del Dolor y Cuidados Paliativos (PAD y CP) fue incorporado al Sistema de Acceso Universal de Garantías Explícitas (AUGE) (3), que ha permitido garantizar plazos máximos para el acceso de las personas con cáncer avanzado a confirmación diagnóstica y tratamiento. La atención de los pacientes con cáncer avanzado se efectúa a través de lo establecido en las Normas Generales Técnicas del Ministerio de Salud Nos 31 (2) y 32 (4), donde están contenidos los protocolos del tratamiento de dolor por cáncer y control de otros síntomas, junto a la atención de Enfermería.

El principal objetivo del Programa, en esta etapa de la enfermedad, es "Mejorar la calidad de vida de los enfermos con cáncer avanzado de ambos sexos y toda edad", a través de la derivación, ingreso, evaluación y tratamiento de la unidad paciente - familia, implementado a través de 28 centros o unidades PAD y CP del adulto a lo largo del país, los que constituyen la red nacional de atención en íntima relación con las unidades de oncología, hematooncología, patología mamaria y cervical, cirugía y otras especialidades de nivel secundario y terciario de atención. También tiene nexos con los laboratorios clínicos, imágenes, centros de radioterapia y con los departamentos de hematología de los 28 Servicios de Salud del país. Desde 1998 financia el 100\% del tratamiento con analgésicos e insumos prioritarios a todos los beneficiarios del sector público. Dispone, desde sus inicios, de registros sistemáticos específicos para el monitoreo, la evaluación y seguimiento de los casos ingresados; sus antecedentes conforman una base de datos nacional.

Hasta la fecha, sólo existen algunos estudios documentados $(5,6)$ sobre las experiencias de los chilenos (y/o sus familias) que acuden a los servicios de cuidados paliativos. Además, existen pocos estudios, en Chile, sobre la experiencia con el fenómeno del proceso de duelo y el proceso de morir (7). Con el interés de profundizar respecto de ambas temáticas que experiencian los pacientes y familiares durante su estadía en el Programa de Alivio del Dolor y Cuidados Paliativos, se realizó este estudio, cuyo propósito fue aumentar la comprensión acerca de cómo los usuarios de cuidados paliativos enfrentan estos cuidados.

El enfrentamiento de los pacientes con el Programa de Alivio del Dolor y Cuidados Paliativos, en la mayoría de las situaciones, se da en forma impensada por los pacientes; es decir, durante su control en el Programa de Cáncer les es informado su ingreso al Programa de Alivio del Dolor y Cuidados Paliativos para un mejor manejo de sus síntomas, dado el avance de su enfermedad, ocurriendo que en ocasiones, los pacientes aún no conocen su diagnóstico (5). Sin embargo, "la situación de enfermedad avanzada implica la presencia explícita de la muerte y una amenaza de pérdida a corto y mediano plazo" (8). El equipo tratante, en esta primera etapa de 
ingreso a la Unidad y al Programa, además de ofrecer un apoyo concreto al paciente y a su grupo familiar, deberá tomar en cuenta su funcionamiento y el proceso de adaptación a la enfermedad. Posteriormente, la persona afectada sigue acudiendo a las citaciones, personalmente $\mathrm{o}$, si su funcionamiento físico o emocional se lo impide, existe la posibilidad que sus familiares asistan, para recibir las indicaciones y el tratamiento del equipo tratante. En ocasiones, es visitado en su domicilio por el equipo de salud del Programa, ya sea el médico, enfermera, trabajadora social, psicólogos y otros (5).

El enfrentamiento de esta serie de situaciones no ha de ser fácil para las personas ni para sus familias, en el fondo constitutivo de situaciones límites o desbordante de su cotidianidad. Para entender esta realidad, la teoría del enfrentamiento de Lazarus y Folkman (9) proporciona una comprensión de las experiencias que las personas con cáncer avanzado y sus familias mencionan en esta etapa crítica de la enfermedad. Estos autores definen enfrentamiento como "aquellos esfuerzos cognitivos y conductuales constantemente cambiantes que se desarrollan para manejar las demandas específicas externas y/o internas que son evaluadas como excedentes o desbordantes de los recursos del individuo" (9).

Referirse al proceso de enfrentamiento en las personas concurrentes a Cuidados $\mathrm{Pa}$ liativos significa referirse al establecimiento de un cambio en los pensamientos y actos en las personas afectadas, a medida que la interacción va desarrollándose; por ende, el enfrentamiento aquí es una experiencia o proceso cambiante en que el individuo, en determinados momentos, debe contar principalmente con estrategias, ya sean defensivas $\mathrm{u}$ otras que le sirvan para resolver el problema; todo a medida que vaya cambiando su relación con el entorno. Las variaciones pueden ser el resultado de los esfuerzos de enfrentamiento dirigidos a cambiar el entorno o su traducción interior, modificando el significado del acontecimiento o aumentado su comprensión. Las etapas de enfrentamiento, mencionadas por Lazarus y Folkman (9) pueden ser, en este caso, el simple reflejo de las demandas físicas o ambientales cambiantes y de las correspondientes secuencias internas que tienen lugar en el sujeto.

\section{MATERIAL Y MÉTODO}

La metodología implementada en este estudio fue el abordaje fenomenológico y exploratorio. Esta metodología tiene como objetivo la comprensión detallada de las creencias, actitudes, valores y motivaciones de las personas en contextos específicos (10); e intenta describir las experiencias de participantes que comparten un fenómeno común. Al mismo tiempo, trata de encontrar la experiencia principal de los participantes a través de un análisis de sus discursos. Esta forma de recolección de información resulta fundamental para poder distinguir la esencia de las experiencias de los participantes con el fenómeno del tratamiento paliativo y la última etapa de la vida.

\section{Diseño del estudio}

Se condujo el estudio a través de la técnica focus groups o grupo focal, por ser más adecuada cuando se busca explorar/ profundizar la discusión y el debate. Se hicieron dos sesiones de focus groups en el Instituto Nacional del Cáncer, en Santiago, Chile. Una sesión fue hecha con un grupo de cinco pacientes que estaban recibiendo tratamiento paliativo en el Instituto y la otra sesión se hizo con cinco familiares que cuidaban o habían cuidado algún familiar que recibía tratamiento paliativo en el mismo Instituto. Entre ellos no había relación de parentesco. Cada sesión duró aproximadamente dos horas.

\section{Recolección de datos}

Los grupos fueron seleccionados previo lla- 
mado voluntario en el Instituto Nacional del Cáncer, mediante avisos. Se seleccionaron a participantes que tenían experiencia necesaria para hablar del tema del cuidado paliativo y habían expresado interés en participar en el estudio. Los focus groups se completaron a través de una serie de preguntas que requerían respuestas amplias. Cada sesión fue realizada por el equipo investigador. Las preguntas realizadas a los participantes durante las sesiones, fueron creadas por el equipo investigador y revisadas por expertos en el tema. Las siguientes preguntas de investigación fueron exploradas durante las sesiones: 1) ¿Cuéntenme sobre sus experiencias con los cuidados paliativos; 2) ¿Cómo definen ustedes calidad de vida?; 3) ¿Qué significado tiene o cómo definen ustedes la palabra dignidad?; 4) ¿Qué significado tiene la palabra autocuidado para ustedes? (esta pregunta fue hecha solamente a los familiares que proveían cuidados).

\section{Análisis de los datos}

Las sesiones de los grupos focales fueron grabadas y transcritas fidedignamente, discursos que fueron analizados, usando el método de Análisis de Contenido (5). Cada transcripción fue leída por lo menos cinco veces, a través de un proceso de asignación de código bilateral. Se utilizaron colores para subrayar palabras claves y términos repetitivos, que se traspasaron a tarjetas (las cuales se usaron para agrupar temas en categorías). Así, se identificaron temas globales (códigos unificados) en los discursos transcritos. Es decir, después de la lectura de cada transcripción, se identificó temas que se repetían, los que se denominaron Códigos Unificados, luego éstos se agruparon en categorías, para finalmente reagruparlos en Códigos Temáticos Finales.

Validación: La validación como elemento de confiabilidad es un aspecto crítico de los estudios cualitativos. En este caso las categorías temáticas identificadas se contrastaron con los mismos participantes de ambos grupos. Se contactó a tres miembros de los grupos, a quienes se les presentó la Categoría Temática Central a través de una entrevista telefónica. Aspectos éticos: A todos los participantes se les informó de los objetivos del estudio y se les solicitó su consentimiento informado. Todos los participantes dieron su consentimiento para participar, junto con otorgar su permiso escrito y oral para que se pudiera grabar las sesiones. Este consentimiento está de acuerdo con las reglas del "Institutional Review Board" (IRB) de la Universidad de Alabama en los Estados Unidos. El Comité de Ética del Instituto Nacional del Cáncer de Chile autorizó la realización del estudio.

\section{RESULTADOS}

Descripción de la muestra: Todos los participantes reportaron haber nacido en Chile y ser católicos. La edad promedio de los pacientes fue de 60 años, y la de las personas que proveían cuidados fue de 49 años; la mayoría de los participantes era casado/a. Todos vivían en diferentes comunas de Santiago. Para los pacientes, el tiempo promedio de estar recibiendo tratamientos paliativos era de 16,2 meses. Para las personas que proveían cuidados, entre los que se encontraban dos varones, quienes eran hijo y esposo, el tiempo promedio de permanencia cuidando a su familiar era de 18,6 meses.

\section{Códigos temáticos que emergieron de los discursos de pacientes y cuidadores:}

De los análisis de los discursos en los focus groups de pacientes y familiares emergieron 24 Códigos Unificados para los pacientes y 25 para los familiares, los que se muestran en Tabla 1: 
Tabla 1: Códigos Unificados de los Pacientes y Familiares

\begin{tabular}{|l|l|}
\hline Códigos Unificados: Pacientes & Códigos Unificados: Familiares \\
\hline 1. Emociones & 1. Aspectos religiosos \\
2. Expresión de Emociones & 2. Aspectos emocionales \\
3. Conciencia personal & 3. Sentimientos \\
4. Intuición personal & 4. Estrés \\
5. Enfrentamiento de situaciones difíciles & 5. Roles de los cuidadores \\
6. Roles de los hombres & 6. Autocuidado \\
7. Roles de los miembros de la familia & 7. Lecciones de la vida \\
8. Roles de los funcionarios de la clínica & 8. Ganancias personales \\
9. Conflicto de roles & 9. Perspicacias nuevas \\
10. Roles de la clínica & 10. Roles de los funcionarios \\
11. Atributos positivos de los funcionarios & 11. Conflicto de roles \\
12. Aprecio & 12. Roles de la clínica \\
13. Autocuidado & 13. Deficiencias \\
14. Calidad de vida & 14. Aspectos financieros \\
15. Dignidad & 15. Desafíos \\
16. Desafíos familiares y personales & 16. Trayectoria del cáncer \\
17. Necesidades de la familia & 17. Necesidades personales \\
18. Preocupaciones financieras & 18. Roles de los vecinos \\
19. Preocupaciones físicas & 19. Aspectos familiares \\
20. Esperanza & 20. Roles de los funcionarios \\
21. Roles de los vecinos & 21. Atributos de la clínica \\
22. La familia & 22. Atributos de los funcionarios \\
23. Religión & 23. Dignidad \\
24. Aspectos espirituales & 24. Necesidades conyugales \\
& 25. Opiniones del paciente \\
\hline
\end{tabular}

Estos Códigos Unificados se agruparon en categorías, desde donde resultaron los Códigos Temáticos Finales de Pacientes y Familiares/Cuidadores, que se muestra en la Tabla 2:

Tabla 2: Códigos Temáticos Finales de Pacientes y Familiares

\begin{tabular}{|l|l|}
\hline Códigos Temáticos Finales: Pacientes & Códigos Temáticos Finales: Familiares \\
\hline 1. Pertenencia personal & 1. Lecciones acerca de cómo cuidar \\
2. Creación de roles en la familia & 2. Clarificación de roles \\
3. Desafíos personales & 3. Enfrentar desafíos \\
4. Raíces psico-espirituales y emocionales & \\
\hline
\end{tabular}

Resultados de Códigos Temáticos Finales de los pacientes:

Código 1: Pertenencia Personal: En este primer Código se incluyeron diferentes tópicos, tales como "espiritualidad, religión, aspectos positivos de los funcionarios del Instituto Nacional del Cáncer, autocuidado, familia y vecinos". Los pacientes mencionaron expresamente su sentido de pertenencia al Instituto 
Nacional del Cáncer. El sentido de apoyo de familiares y de vecinos contribuye a estos sentimientos, no sólo al Instituto, sino también a un núcleo familiar y comunitario. Al respecto, Isabel expresa su deseo de vivir por su familia: "Tengo un sentimiento de continuar, salir adelante, deseo vivir...para mi esposo, mis hijos y mi familia". Las experiencias que los pacientes han tenido dentro del Instituto, les han dado herramientas como para sentirse parte no sólo de la institución sino que también han creado raíces, que les permiten tener un balance emocional en sus vidas. Los funcionarios proporcionan atención integral, acompañan, acogen e intentan comprender el sufrimiento de los pacientes, lo que combinado con un sentido espiritual y conexión a un ser supremo, contribuyen al sentido de pertenencia personal no sólo al interior del Instituto sino también en el mundo externo del paciente.

\section{Creación de roles dentro de la familia:}

Este código temático es definido en términos de un intercambio con el medio ambiente del paciente. Isabel expresa: "Uno es como el dueño de casa aquí...cuando llegas te tratan diferente...somos dueños de casa". A través de esta opinión, se puede ver que la disposición (del Instituto Nacional del Cáncer) con funcionarios, familia del paciente y sus vecinos, contribuyen al desarrollo de roles específicos. Los datos revelaron que algunos de estos roles son difíciles de asumir. Este proceso de cuidar y sus desafíos ha sido mencionado también en otros estudios del tema $(11,12$, 13). La mayoría de los participantes en este estudio reportaron que han asumido roles duales, como el caso de Rosa, quien es una paciente enfrentando un cáncer avanzado y al mismo tiempo cuida a sus nietas. Muchos de los participantes hablaron sobre lo difícil que es asumir este doble papel. Además, hablaron sobre "lo que podíamos hacer antes y después de la enfermedad”. Este conflicto de roles fue expresado en términos de desamparo y dependencia de otros.
Los participantes mencionaron los roles de género que se han desarrollado a través de sus condiciones terminales. Están los estereotipos, Isabel dice: "Los hombres no están preparados para las responsabilidades que requiere el cuidado de una persona terminal". Lo que sin embargo se desmiente con la presencia de dos varones que llevaban ya 18 meses cuidando a su familiar.

Carmen, Ana y Rosa expresaron el desarrollo de resistencia emocional que ellas han debido crear, ya que se han visto forzadas a ser independientes en su autocuidado.

3. Desafíos personales: Los códigos unificados en este tema incluyen: "Deseos, orientación del futuro, asuntos físicos, necesidades personales, desafíos, asuntos financieros y enfrentar temas personales y familiares". La mayoría de los desafíos personales reportados tienen que ver con aspectos de ajuste físico y psicológico, como la declinación de la salud y del funcionamiento físico. También identificaron contenidos de duelo asociados a: pérdida de salarios; del rol de contribución a la familia; y duelo anticipado de las personas que proveen cuidados paliativos. Los desafíos al enfrentar la vida después del diagnóstico, son aceptar cambios físicos y del estado de vida. Pero, como se explicó previamente, ellos mencionaron que ha sido importante para ellos tener la motivación y la actitud positiva de sus familias, comunidad y a los funcionarios del Instituto.

\section{Raíces psico-espirituales y emocionales:} Articulan su sentido de dignidad, espiritualidad e identificación, expresando sentimientos que han emergido a través de la evolución de su enfermedad. Identifican sentimientos de pena, angustia, soledad, estrés, amor y de no tener esperanzas, definiendo conceptos como religión y espiritualidad. Isabel, por ejemplo, se enfocó en su sentido de espiritualidad y su conexión con un "Ser Supremo"; Ana habló de su conexión con "Dios" y "su poder"; Rosa mencionó su sentido de espiritualidad. Esta 
discusión llevó al grupo a hacer una relación con la dignidad: Ana identificó que la dignidad significa: "Hacer el bien a otros" y "Ser honesto porque Dios nos está mirando". Otros mencionaron la calidad de vida y cómo ellos la consideraban estrechamente relacionada con la dignidad. Todos los pacientes estuvieron de acuerdo que para ellos calidad de vida significa: "Estar consciente y atento a lo que está ocurriendo alrededor de uno". También concordaron en que solamente ellos pueden definir lo que es la dignidad y hablaron sobre la importancia de preguntar a la gente sobre sus definiciones de dignidad.

\section{Resultados de códigos temáticos en personas que proveen cuidado:}

1. Lecciones acerca de cómo cuidar: Este código temático fue discutido y presentado por ellos en forma positiva y con desafíos. Por ejemplo, Elena identificó que el cuidar "ha juntado a la familia mucho más...estamos más unidos". También, el grupo habló sobre sistemas de apoyos informales, los cuales se desarrollaron a través de sus experiencias como personas suministrando cuidado. Otro tema identificado fue el aprendizaje de cómo cuidar. Estas destrezas les han sido enseñadas por enfermeras y médicos, lo que les ha permitido ahorrar dinero para sus familias. Elena y Norma hablaron sobre su autoestima cuando aprendieron lo necesario para proveer cuidado a sus familiares enfermos. Aprender a cuidar a un familiar con una condición terminal les ha permitido jugar diferentes roles que no creían poder hacer.

Juan y Norma hablaron sobre los desafíos en el área económica ya que Juan, por ejemplo, estaba desempleado y no podía "contribuir a la familia”. Este tema fue conectado con una discusión sobre el autocuidado y poder hacer las cosas que ellos hacían antes. Las limitaciones de los familiares enfermos han llevado a las personas que proveen cuidados a sentirse sumamente responsable de ellos. Norma compartió que se siente "Amarrada porque no puedo ir a ningún lado sola”. Identificaron estrés, por trabajar periodos tan largos proveyendo cuidados sin tener un descanso. Como dijo Juan: "Es todos los días, lunes a domingo, cada hora y minuto... no hay un descanso". Esta carga emocional de cuidar a un enfermo, y sus efectos como el estrés y depresión, lo menciona también Douglas, Daly, Kelly y Montenegro (14); y Christakis y Allison (15). Coincidentemente, también reportaron que sienten una forma de refugio en el Instituto Nacional del Cáncer. Como dijo Norma: "Uno se siente como si perteneciera aquí". Aparentemente, el tratamiento y el respeto de los funcionarios del Instituto han sido factores valiosos para disminuir el nivel de estrés de las personas que proveen cuidados en esta etapa. Como dijo Juan y el grupo estuvo de acuerdo: "El tratamiento profesional empieza en la puerta del Instituto Nacional del Cáncer y sigue para adentro". Este sentido de "pertenecer" es mencionado en el estudio de Coristine et al. (11), los cuales encontraron que los cuidadores, en su estudio, valorizaron la comunicación de los funcionarios y la relación profesional de los funcionarios, lo que aumentó el nivel de autocontrol descrito por estos cuidadores.

Una lección aprendida ha sido la conciencia de qué significa "calidad de vida". Juan compartió que es "estar consciente, saber y tener la habilidad física de hacer cosas". O, que está asociada a la duración de la enfermedad. Es decir, mientras más larga la enfermedad menos calidad de vida existe. El tema de la dignidad fue presentado por ellos refiriéndose al tratamiento digno entregado por los funcionarios del Instituto a pacientes y familiares.

2. Clarificación de roles: Este fue el segundo tema que emergió de los discursos del grupo. El grupo indicó que los diferentes roles de personas que proveen cuidados se han desarrollado a través de una combinación de factores. Los participantes identificaron lo importante que ha sido el rol de los funcionarios del Instituto en ayudarlos a establecer 
y clarificar sus roles como personas que proveen cuidados. Un ejemplo fue presentado por Elena cuando habló de las enseñanzas y la tranquilidad que la enfermera le otorgó cuando cuidaba a su tío.

Juan habló sobre sus experiencias y dificultades en asumir su rol. Específicamente, Juan habló sobre el asunto de la intimidad sexual con su esposa: "Nosotros no hemos tenido relaciones por dos años".

El grupo habló con gran detalle sobre la importancia de tener el apoyo familiar cuando estos roles se desarrollan. Específicamente, el grupo habló del apoyo de los vecinos, la familia, y los funcionarios del Instituto. Juan, Norma y Luisa compartieron cuánto han valorado la información y la guía que el Instituto les ha ofrecido con el manejo de medicinas. Los participantes también hablaron del rol principal de la persona que provee cuidado y algunos participantes, como Luisa y Elena, creen que este rol tiene que ser compartido entre la familia, pero Norma añadió que "el cuidado tiene que ser supervisado solamente por una persona".

3.Enfrentar desafíos: Es la última categoría que emergió en el análisis. Muchos de los asuntos en este tema se tocaron en otras áreas o discusiones en el grupo. Sin embargo, uno de los temas confluentes fue el asunto de la dependencia del paciente. Todos identificaron que la dependencia del paciente se traduce en independencia limitada para las personas que proveen cuidados. Este fenómeno parece tener un impacto directo en el autocuidado de las personas que proveen cuidados. Todos concordaron en el cansancio físico que han experimentado debido a los largos periodos siendo personas que proveen cuidados. Sin embargo, el cansancio emocional fue comentado mucho más en el grupo. Este cansancio emocional emerge cuando las personas que proveen cuidados sienten culpabilidad de no poder dar más de lo que pueden. Juan identificó su nivel de frustración y cansancio cuando dijo: "A veces uno se cansa... a veces uno está harto de dar este cuidado todos los días".

Otros desafíos expresados por el grupo fueron en el área de la infraestructura general. Comentaron sobre los pasillos llenos de pacientes y familiares. Andrés extrapoló sobre esto: "Yo creo que hay algo detrás de lo que estamos hablando... a lo mejor hay esa amabilidad que ustedes han identificado... pero llegar acá significa pasar por calles que tienen muchos problemas... hay muchos autos... hay muchos hoyos... a lo mejor si uno no está enfermo no se da cuenta de estas cosas".

\section{DISCUSIÓN}

Al principio del análisis se consideraba extraer dos distintos códigos temáticos centrales, derivados de ambos grupos. Sin embargo, el análisis arrojó muchas similitudes, comprendiendo que existía en el grupo sólo un código temático central, al que confluía cada uno de los códigos identificados, que describía las experiencias de los participantes de los dos grupos. Emerge así la idea que vivenciar las experiencias de una enfermedad terminal y cuidados paliativos cambiaron la vida de los participantes. Los participantes estaban describiendo sus experiencias con una conexión a algo mayor, lo cual ha sido previamente mencionado en el estudio de Coristine et al. (11). La mayoría de los participantes hablaron sobre sus relaciones con vecinos, médicos, enfermeras, trabajadoras sociales y otros pacientes. Estas relaciones, como fueron discutidas en los grupos, se desarrollaron a través del proceso de enfrentar una enfermedad terminal. Lo que parece tener en común los dos grupos y unirlos es pertenecer a algo mayor.

La solidaridad construida por ambos grupos se verifica cuando los datos de ambos grupos se analizan conjuntamente. Los participantes expresaron esta solidaridad cuando hablan sobre una esencia de santuario. Este santuario fue descrito por los participantes en 
forma estructural, es decir, de cómo se sentían en relación con la gente, al tema psicosocial y a la estructura física del Instituto Nacional del Cáncer. Es decir, la experiencia personal por la cual han pasado les ha permitido la creación de un santuario en donde existe una comunión entre lo personal y lo espiritual. Este santuario ha ayudado a ambos grupos a enfrentar con dignidad y calidad de vida la difícil etapa creada por esta enfermedad terminal. La palabra "santuario" se usa en este estudio con un significado de trascendencia desde un mínimo nivel de conocimiento sobre el propósito que uno tiene en la vida, a otro en donde existen nuevos conocimientos y experiencias emocionales, espirituales y muchas veces físicas. Esto hace que la percepción sensorial (audición, olfato, gusto, visión, tacto) aumente y la persona desarrolle una nueva y consciente apreciación del mundo. Por lo tanto, las experiencias de los pacientes y personas que proveen cuidados paliativos se puede resumir con un único Código temático central, identificado en este estudio como pertenecer a algo mayor. Cuando el Código temático central le fue leído a una de las participantes, Isabel expresó: "Esta frase tiene un significado muy profundo para mí y describe mucho de mi vida... las cosas cambian cuando uno se enferma y algo importante es poder encontrar ese refugio, ese santuario".

Otro participante, Adrián, fue contactado para validar los códigos. Cuando las palabras "refugio" y "santuario" fueron identificadas, Adrián dijo: “'Sí!, son importantes para mí... tener el apoyo del Instituto Nacional del Cáncer y nuestros amigos... si no fuera así estaríamos solos, sin el apoyo moral y sin el apoyo espiritual que tanto necesito". La contribución de Adrián sobre el "refugio" y "santuario" es importante ya que él contextualiza el significado de estos temas. Está claro que para Adrián encontrar este sentido de refugio y santuario es un evento externo. Para Adrián, santuario es otorgado por otras personas y por los servicios que reciben, él y su esposa, en el Instituto Nacional del Cáncer.
Esta interpretación plantea a los equipos tratantes la necesidad de socializar con la enfermedad en el modo de sentirla y vivirla. La comprensión psicológica del paciente y el deseo de penetrar hasta los problemas emocionales íntimos del paciente, debería formar parte del arsenal terapéutico del equipo tratante y de su entorno más íntimo.

La esencia, o el código temático central de este estudio permitiría entender que los sujetos sociales construyen significados desde su propio mundo. También colige que transmitir al paciente que hay un tiempo disponible para escucharlo, sin bloquear sus discursos, sin juzgar, usando un lenguaje cálido y cómplice, coadyuvará a mantener un visión positiva, humanista, de los equipos tratantes de cuidados paliativos, lo cual concuerda con lo que señalan Middleton (16) y Rosdale (17). Esto también está relacionado con lo aportado por Lazarus y Folkman (9) sobre los procesos de enfrentamiento: significan una experiencia de cambio en los pensamientos y actos a medida que la interacción va desarrollándose; por ende, esta experiencia que produce cambios en el individuo debe contar con estrategias, ya sean defensivas u otras que sirvan para resolver el problema (implican apoyos externos), todo a medida que vaya cambiando su relación con el entorno. "Se debe tener en cuenta que hoy en día en nuestra sociedad se produce un individualismo que lleva a una sensación general de alineación y desconexión, que impide la formación de un sentido de pertenencia de carácter colectivo y de un sistema de apoyo en red" (6). Así el grupo percibe un sentido de pertenencia, por lo que se puede concluir que ellos han encontrado en el equipo tratante, consuelo, apoyo, confianza, entrega de sugerencias de orden práctico y una atención integral. Esta cercanía con el equipo de salud ha sido identificada también por investigaciones (18), agregando elementos afrontativos positivos. El acompañamiento, por lo tanto, será clave en la relación equipo tratante-familia-paciente, pues en este proceso de la enfermedad, éstos 
sienten la necesidad de estar acompañados, la soledad es muchas veces peor que la muerte, es por ello la necesidad de estar donde haya calidez y afecto en las relaciones con los otros, que les permita estar seguros en su hábitat.

Cobra relevancia el trabajo en equipo interdisciplinario, como lo señalan Reyes, Santi y Nervi (19),"Modelos de cuidados paliativos multidisciplinarios se necesitan en la formación de los profesionales de la salud, para expandir el crecimiento de la atención multidisciplinaria orientada al paciente y a las familias a lo largo del país". En esa perspectiva, Espinoza y Sanhueza (7) señalan: "Enfermería por su formación humanística tiene el desafío de gestionar una atención de calidad, lo cual trae consigo la necesidad de incrementar su preparación en esta área y generar un mayor número de evidencia en la misma, que propenda a una mejor calidad del morir en la persona con cáncer". De allí que, se hace cada vez más comprensible la inclusión de distintos profesionales en los programas de cuidados paliativos en Chile, como parte integrante de dicho programa, como sería el caso de los Trabajadores Sociales. Las miradas holísticas de las distintas disciplinas coadyuvarán al desarrollo de un trabajo en equipo y permitirá tener una visión integral en cada situación.

Existen otras áreas en que es necesario realizar investigaciones cualitativas, de parte del equipo, que incluyen el cuidado de pacientes: el autocuidado del paciente mismo y el autocuidado de los profesionales que proveen cuidados paliativos.

Se necesitan además investigaciones en todos los aspectos biosico-socioespiritual de los cuidados paliativos en Chile. Incorporar el aspecto "espiritual" de la persona promueve la evaluación holística (7) y Breitbart, Gibson, Poppito y Berg (20) lo incluyen en los servicios que se les ha de brindar a estas familias. Cobra igualmente importancia otorgar asistencia psicosocial a los familiares después de la muerte de un ser querido. Lo manifestaba Kúbler-Ross (21): "Lo más importante es nuestra propia actitud y nuestra capacidad para afrontar la enfermedad mortal y la muerte. Si éste es un gran problema en nuestra vida, y vemos la muerte como algo tabú, aterrador y horrible, nunca podremos ayudar a un paciente a afrontarla con tranquilidad". La comprensión del concepto de calidad del morir según lo anterior y lo que se desprende del estudio implica que la persona se encuentra consciente de su muerte próxima, logrando un sentido de control para él o ella y respetando sus deseos y de sus cercanos (22).

Es tal la importancia de los cuidados paliativos, que en la actualidad muchos países propician la inclusión de contenidos paliativos en el diseño curricular de las distintas profesiones de la salud en pregrado. La intervención en el duelo ha sido incorporada como un servicio de forma rutinaria en otros países como Inglaterra, Estados Unidos y Canadá. Recomendable sería crear algunos focus groups con personas que han pasado por este duelo y tratar de reconceptualizar sus experiencias. Estas conllevarían directamente a la evaluación de las necesidades y a la creación de nuevas intervenciones. Como crear un modelo de terapia familiar protocolizado, con profesionales capacitados en proveer una atención adecuada a las necesidades en el proceso de duelo. Otras investigaciones en el futuro pueden usar los resultados de este estudio exploratorio para desarrollar nuevas preguntas que se enfoquen en cuidados paliativos, el duelo anticipatorio, la dimensión sexual, etc., para identificar las necesidades de pacientes y personas que proveen cuidados paliativos. Crear un mapa comunal, que identifique personas que proveen cuidados puede ayudar a conectar a familias con ciertos recursos dentro de sus propias comunidades. Se recomienda utilizar la evidencia de estudios cualitativos para capturar las experiencias cotidianas de esta población. También es esencial que la red profesional médica tenga en cuenta las necesidades bio-psicosociales de pacientes, sus cuidadores y seres queridos, que enfrentan tratamientos paliativos 
y condiciones terminales. Finalmente, se desprende la importancia de confrontar las necesidades bio-psicosociales identificadas por pacientes, seres queridos y equipo multi/ inter-disciplinario, identificado también en otros estudios (23-25).

\section{REFERENCIAS}

1. Chávez, Cura, Derio, Guerrero, Nervi, Reyes. "Symptom control and palliative care in Chile". Journal of Palliative Care and Pharmacotherapy. 17: 13-22; 2003.

2. MINSAL. Ministerio de Salud de Chile. Norma General Técnica No 32: Alivio del Dolor por Cáncer y Cuidados Paliativos; 1999.

3. AUGE- Protocolo de trabajo (2004). Revisado el 01/07/2008, en http://www.minsal. cl/juridico/DTO_228_AUGE_2006.doc

4. MINSAL. Ministerio de Salud de Chile. Norma General Técnica N³1: Norma de Enfermería Alivio del Dolor por Cáncer y Cuidados Paliativos; 2002.

5. Sanhueza O, Valenzuela S, Barriga O, Espinoza M, Torres A, Stiepovich J, Paravic T. Informe FONIS- Conicyt-No SA 05I20022 "Calidad de vida de usuarios de programas de Cuidados Paliativos", Concepción, Chile; 2007.

6. Cancino $G$, Las vivencias de lo cotidiano en pacientes oncológicos - La mirada de Trabajo Social. Tesis magíster en Trabajo Social, Universidad Academia de Humanismo Cristiano (UAHC), Santiago, Chile; 2006.

7. Espinoza M, Sanhueza O, Contribuir a la calidad del morir en el cáncer. Ciencia y Enfermería XIII (2): 17-23. Chile; 2007.

8. Juvero M. Reflexión sobre el Trabajo Social en Cuidados Paliativos. Lérida, España; 2006.

9. Lazarus R, Folkman S. Estrés y procesos cognitivos ( $2^{\mathrm{a}}$ ed.) 124-252. Barcelona. España. Ed. Martínez Roca; 1986.
10. Minayo MC de S. O desafio do Conhecimento: Pesquisa qualitativa em saúde. $8^{\mathrm{a}}$ ed. São Paulo, Hucitec; 2004.

11. Coristine M, Crooks D, Grunfeld E, Stonebridge C, Christie A. Caregiving for women with advanced breast cancer. Psycho-Oncology, 12; 2003.

12. Weiss CO, González HM, Kabeto MU, Langa KM. Differences in amount of informal care received by non-hispanic whites and latinos in a nationally representative sample of older americans. Journal of the American Geriatrics Society. 53; 2005.

13. Navaie-Waliser M, Feldman PH, Gould DA, Levine C, Kuerbis AN, Donelan $\mathrm{K}$. The experiences and challenges of informal caregivers: Common themes and differences among whites, blacks, and hispanics. Gerontologist, 41; 2001.

14. Douglas SL, Daly BJ, Kelly O’Toole E, Montenegro H. Impact of a disease management program upon caregivers of chronically critically ill patients. CHEST, $128 ; 2005$.

15. Christakis NA, Allison PD. Mortality after the hospitalization of a spouse. The New England Journal of Medicine. 354; 2006.

16. Middleton J. Acompañamiento psicológico al paciente en etapas avanzadas de enfermedad. Monografía; 2004.

17. Rosdale M. Survivor loneliness of women following breast cancer. Oncology Nursing Forum 36; 2009.

18. Gerd D, Marit S, Jordoy MD, Kaasa S. Family Satisfaction with End-of-Life Care for Center Patients in a Cluster Randomized Trial. J Pain Symptom Managet 2002 July; 24(1) 53-63.

19. Reyes, Santi, Nervi. Cuidados Paliativos y control de síntomas en Chile. La experiencia de clínica familia. Rev ARS Médica, v(11), No 11. U. Católica, Chile; 2004.

20. Breitbart W, Gibson C, Poppito S R, Berg A. Psychotherapeutic Interventions at the end of life: a focus on meaning and spirituality. Canadian Journal of 
Psychiatry, 49, 366-372; 2004.

21. Kübler-Ross E. The Languages of dying. Journal of Clinical Chile Psychology 3:22-24 En Cuidados Paliativos: Atención Integral a Enfermos Terminales Vol.II Ed. ICEPSS, España. 1974.

22. Patrick D, Engelberg R, Curtis R. Evaluating the Quality of Dying and Death. Journal of Pain and Symptom Management 22 (3): 717-726; 2001.

23. Demmer C. A national survey of hospice bereavement services. Omega, 47; 2003. 24. Newell S, Sanson-Fisher RW, Girgis A, Bonaventura A. How well do medical oncologists' perceptions reflect their patients' reported physical and psychosocial problems? - data from a survey of five oncologists. Cancer, 83; 1998.

25. Kelly BJ, Turner J. Depression in advanced physical illness: diagnostic and treatment issues. Medical Journal of Australia, 190; 2009. 